\title{
Characterization of pyridomycin B reveals the formation of functional groups in antimycobacterial pyridomycin
}

1 Tingting Huang ${ }^{*}$, Zihua Zhou, Maolong Wei, Lin Chen, Zhihong Xiao, Zixing Deng, Shuangjun Lin

\section{Affiliations}

5 State Key Laboratory of Microbial Metabolism, and Joint International Research Laboratory on

6 Metabolic\& Developmental Sciences, and School of Life Sciences \& Biotechnology, Shanghai Jiao

7 Tong University, Shanghai, 200240, China

9 * Address correspondence to Tingting Huang, thingwing82@ @jtu.edu.cn

10 Keywords: pyridomycin, antimycobacterial activity, biosynthesis, enoyl ester, trans ketoreductase

11 Running title: Characterization of the new pyridomycin analogue 


\section{Abstract}

14 Pyridomycin, a cyclodepsipeptide with potent antimycobacterial activity, specifically inhibits the

15 InhA enoyl reductase of Mycobacteria tuberculosis. Structure-activity relationship studies indicated

16 that the enolic acid moiety in pyridomycin core system is an important pharmacophoric group and the

17 natural configuration of the $\mathrm{C}-10$ hydroxyl contributes to the bioactivity of pyridomycin. The ring

18 structure of pyridomycin was generated by the nonribosomal peptide synthetase (NRPS) and

19 polyketide synthase (PKS) hybrid system (PyrE-F-G). Bioinformatics analysis reveals that SDR

20 family protein Pyr2 functions as a 3-oxoacyl ACP reductase in the pyridomycin pathway.

21 Inactivation of pyr2 resulted in accumulation of pyridomycin B, a new pyridomycin analogue

22 featured with enol moiety in pyridyl alanine moiety and a saturated 3-methylvaleric acid group. The

23 elucidated structure of pyridomycin B suggests that rather than functioning as a post-tailoring

24 enzyme, Pyr2 catalyzes ketoreduction to form the C-10 hydroxyl group in pyridyl alanine moiety and

25 the double bond formation of the enolic acid moiety derived from isoleucine when the intermediate

26 assembled by PKS-NRPS machinery is still tethered to the last NRPS module, in a special energy-

27 saving manner. Ser-His-Lys residues constitute the active site of Pyr2, which is different from the

28 typically conserved Tyr based catalytic triad in the majority of SDRs. Site-directed mutation

29 identified that His154 in the active site is a critical residue for pyridomycin B production. These

30 findings will improve our understanding of the pyridomycin biosynthetic logic, identify the missing

31 link for the double bound formation of enol ester in pyridomycin and enable creating chemical

32 diversity of pyridomycin derivatives.

\section{Importance}

35 Tuberculosis (TB) is one of the world's leading causes of death by infection. Recently, pyridomycin,

36 the antituberculous natural product from Streptomyces has garnered considerable attention for being 
37 determined as a target inhibitor of InhA enoyl reductase of Mycobacteria tuberculosis. In this study,

38 we report a new pyridomycin analogue from mutant HTT12, demonstrate the essential role of a

39 previously ignored gene pyr2 in pyridomycin biosynthetic pathway, and imply that Pyr2 functions as

40 a trans ketoreductase (KR) contributing to the formation of functional groups of pyridomycin utilize

41 a distinct catalytic mechanism. As enol moiety are important for pharmaceutical activities of

42 pyridomycin, our work would expand the understanding the mechanism of SDR family proteins and

43 set the stage for future bioengineering of new pyridomycin derivatives. 


\section{Introduction}

47 Tuberculosis (TB) remains a major global health problem, causing an estimated 10.0-million people

48 developed TB disease in 2017 (1). With the appearance of multidrug-resistant strains of TB to all the

49 antitubercular chemotherapies, more efficient TB control call for the development of new

50 antimycobacterial drugs. Classic biochemical approaches, '-omics' technologies including genomic-

51 and proteomic-based approaches combined with computational chemistry strategies allow target

52 discovery of natural products with antimycobacterial activity (2-4). Recently, reappraisal of known

53 natural products becomes a research strategy to accelerate the development of antimycobacterial drug

54 leads from natural products $(5,6)$. In Mycobacteria tuberculosis $(M t b)$, InhA is an NADPH-

55 dependent enoyl-acyl carrier protein (ACP) reductase from the FASII pathway essential for

56 synthesizing mycolic acids of cell wall $(7,8)$. The clinical TB drugs isoniazid and ethionamide are

57 prodrugs that are activated by the catalase-peroxidase KatG of $M t b$ to form an adduct with $\mathrm{NAD}^{+}$and

58 then target the substrate-binding site of InhA (9). In the aim of finding new antimycobacterial

59 compounds, pyridomycin has been identified as an InhA competitive inhibitor (10).

60 Pyridomycin (Figure 1A, 1) is a cyclodepsipeptide produced by Streptomyces pyridomyceticus

61 NRRL B-2517 (11). The 12-membered core ring is composed of two pyridyl groups (a N-3-

62 hydroxypicolinyl-L-threonine and a 3-(3-pyridyl)-L-alanine), a propionic acid, and a structurally

63 unique 2-hydroxy-3-methylpent-2-enoic acid (12). From the X-ray crystallography of pyridomycin-

64 InhA complex, the two pyridyl groups of pyridomycin occupy the NADPH cofactor binding site of

65 InhA, and the enolic acid (2-butan-2-ylidene) moiety protrudes into the lipid substrate binding

66 pocket. The unique configuration that simultaneously spans both the cofactor and the substrate-

67 binding pocket of InhA endows pyridomycin with potent activity against InhA-resistant strains of $68 M t b(10,13)$. 
Further structure-activity relationship (SAR) investigation of chemical synthesized

dihydropyridomycins (Figure 1A, 2 and 3) revealed that substitution of the enolic acid moiety of pyridomycin by $2 R$-isopropyl moiety (2) made the compound less active (four-fold lower than pyridomycin) while the substitution with a $2 S$-isopropyl residue (3) led to diminished activity compared to pyridomycin (14). Meanwhile, the hydroxyl group on C-10 is an essential stereocenter

(16) and the establishment of enolic acid moiety was one of the major difficulties. Motivated by the promising activity and the structural uniqueness, we set out to decipher the biosynthesis of pyridomycin both at the genetic and the biochemical level.

Nonribosomal peptide synthetases (NRPSs) and polyketide synthases (PKSs) multifunctional enzyme complexes composed of successive catalytic domains that are themselves coordinated organized into biosynthetic modules. Each module in the assembly line performs iterative chemical reactions to produce numerous natural products (17). In contrast to the co-linear manner of PKSs or NRPSs, a growing number of trans-acting units has been identified, including trans-acyltransferase (AT) for loading PKS building blocks, trans-enoylreductases (ER) for reduction of $\alpha, \beta$-unsaturated intermediates, and trans-ketoreductase (KR) to reduce a $\beta$-ketone to a hydroxyl group (18-23).

85 Previous biosynthetic studies showed that the pyridomycin core structure was assembled by a hybrid domain and a PCP respectively, which formed the loading module to activate and load starter unit 3HPA (3-Hydroxy pyridine carboxylic acid) (24). PyrE, F, G encode NRPS-PKS proteins comprising

90 four modules in total. PyrE is a typical NRPS dimodule consisting of two minimal NRPS modules

91 (C-A-PCP) that are predicted to sequentially incorporate threonine and 3-(3-pyridyl)-L-alanine. PyrF

92 is the sole PKS module (KS-AT-MT-ACP) and was believed to incorporate propionic acid into the 
93 pyridomycin backbone. PyrG is a special NRPS module, which contains two tandem A domains and

94 a PKS-type ketoreductase (KR) domain (25). The second A domain in PyrG was functional for 95 recoganization and activation of $\alpha$-keto- $\beta$-methylvaleric acid (2-KVC) as the native substrate. The 96 KR domain of PyrG further catalyzed the reduction of the 2-KVC tethered onto the PCP of PyrG to 97 generate the PCP-tethered $\alpha$-hydroxy- $\beta$-methylvaleric acid (2-HVC). A biosynthetic pathway has been proposed for the pyridomycin core structure based on these PKS/NRPS related enzymes (24). In addition, there are some other genes in the cluster with yet unassigned biosynthetic functions, which might be involved in the modification step during or after the NRPS/PKS process. thought to be responsible for the reduction of the $\beta$-keto group to form the hydroxyl group $(*)$.

103 Besides, little was known about the formation of double bond of the enolic acid moiety. Close 104 inspection of the pyridomycin gene cluster showed that pyr2 encodes a 3-oxoacyl-acyl-carrier-protein 105 (ACP) ketoreductase that is highly homologous to FabG from the bacterial type II fatty acid synthase.

106 FabG is a member of the ketoacyl reductase family of proteins and is an essential enzyme for type II 107 fatty acid biosynthesis that catalyzes an NADPH-dependent reduction of $\beta$-ketoacyl-ACP to the $\beta$ 108 hydroxyacyl isomer, which is the first reductive step in the elongation cycle of fatty acid 109 biosynthesis. We speculate that Pyr2, a 3-ketoacyl-ACP reductase may act as a freestanding 110 ketoreductase in a similar manner as FabG, to reduce the $\beta$-ketoacyl intermediates tethered to PyrF111 ACP (Figure 1B). By using gene inactivation and complementation, we identified pyr2 as an 112 essential gene for pyridomycin biosynthesis; isolation and characterization of the resulting new 113 pyridomycin derivative from the pyr2 mutant shed new insight into the biosynthesis of functional 114 groups in pyridomycin. Our study not only confirmed a trans-acting NRPS/PKS specific tailoring 115 enzyme creating chemical diversity of pyridomycin, but also provides the opportunity to explore the 116 biosynthetic mechanism of functional moieties in pyridomycin. 


\section{Results}

\section{Bioinformatics analysis of Pyr2}

120 Pyr2, encoding a 3-ketoacyl-acyl-carrier protein reductase, located upstream of pyridomycin NRPS-

121 PKS genes which was originally identified as the boundary of pyridomycin gene cluster (24). Pyr2

122 belongs to the short-chain dehydrogenase/reductases (SDR) super family oxidoredutase with

123 significant sequence identity $(\sim 50 \%)(26,27)$. The amino acid sequence of Pyr2 was blast searched in

124 microbial genome database and compared for its similarity. It reveals that Pyr2 shares sequence

125 homology with 3-oxoacyl-ACP reductase from Streptomyces gancidicus (58\% identity and $70 \%$

126 similarity) or from Allonocardiopsis opalescens (57\% identity and $72 \%$ similarity). A database

127 search of Pyr2 revealed homologs from various microorganisms, mostly are from high GC gram-

128 positive strains in the phylogenetic survey. A sequence similarity network of Pyr2 with its homologs

129 found at an e-value threshold of $10^{-80}$ was established (Figure 2). In the type II FAS system, 3-

130 oxoacyl-ACP reductase catalyzes the first reduction step that leads to the conversion of 3-oxoacyl-

131 ACP to 3-D-hydroxyacyl-ACP intermediate during the elongation cycle. Therefore, Pyr2 was

132 considered as the most likely candidate to catalyze the C-10-keto reduction step in pyridomycin

133 biosynthesis.

\section{Inactivation and Functional Complementation of pyr2}

135 To assess the necessary of $p y r 2$ for pyridomycin production, gene inactivation was achieved

136 through substituting pyr2 by the kanamycin resistance cassette using the $\lambda_{\text {RED-mediated PCR- }}$

137 targeting mutagenesis. The resultant double-crossover mutant S. pyridomyceticus HTT-12 was

138 confirmed and its fermentation profile was detected by HPLC-MS (24). The mutant HTT12 retained

139 the ability to produce a peak with the similar retention time ( 16.4 min) to that of pyridomycin on 
140 HPLC profile, exhibiting a molecular ion peak at $m / z 541[\mathrm{M}+\mathrm{H}]^{+}$same as that of pyridomycin

141 (Figure 3A). The crude extracts of $S$. pyridomyceticus and HTT12 mutant were tested for 142 antimycobacterial activity using a standard disk diffusion assay. Both of them showed similar 143 inhibition zone on the bioassay agar plate (Figure 3B and 3C) which seems that pyr2 is not included 144 within the pyridomycin biosynthetic gene cluster (24).

145 Surprisingly, although the molecular weight of the product from HTT12 is as the same as that of 146 the pyridomycin, the peak from HTT12 does not show a higher absorption shoulder at around $230 \mathrm{~nm}$ 147 from the UV/Vis spectroscopy, which is slightly different from the UV/Vis spectrum feature of 148 pyridomycin (Figure 3B and 3C). A closer inspection and comparison of the LC-MS/MS profile 149 revealed that they had different MS/MS patterns (Figure 3E and 3F). Analysis of the peak from 150 HTT12 in positive-ion mode showed a MS/MS fragmentation pattern $(\mathrm{m} / \mathrm{z} .205 .0,409.1$, and 427.1

151 for $\left.[\mathrm{M}+\mathrm{H}]^{+}\right)$, presenting a -2 mass shift, compared to the fragmentation data $(\mathrm{m} / z, 207.1$, 411.1, and 152429.1 for $[\mathrm{M}+\mathrm{H}]^{+}$) of pyridomycin. Additionally, the two profiles also share the same ion peaks 153 such as $m / z$ at $223.0[\mathrm{M}+\mathrm{H}]^{+}$and $337.0[\mathrm{M}+\mathrm{H}]^{+}$. The assignment of these fragmental ions based on 154 the MS/MS2 data suggests that the peak in HTT12 contains a partial structure of pyridomycin and 155 these fragments with modification on 3-(3-pyridyl)-L-alanine moiety.

156 To test whether the retention time shift and MS/MS pattern variation in HTT12 were actually 157 caused by the in vivo deletion of pyr2, a complementation experiment was carried out through 158 introduction of the integrative vector pJTU4656, a shuttle plasmid harboring a copy of pyr2 under the 159 control of the strong promoter PermE*, into the $\Delta p y r 2$ mutant HTT12 through conjugation. 160 HTT12/pJTU4656 was selected in the presence of both apramycin and kanamycin. Subsequently, the 161 culture extracts from the complementation strain were analyzed by HPLC-MS (Figure 3A, lane c). 162 Although it was relatively less than the wild type strain, the peak in HTT12/pJTU4656 showed same 163 MS/MS pattern as pyridomycin, indicating that pyridomycin production was restored with intact pyr2 
164 complementation. Taken together, the requirement of pyr2 in the biosynthesis of pyridomycin was

165 supported by pyr2 inactivation and complementation. Pyr2 should play a specific role in the biosynthesis of pyridomycin.

\section{Isolation and structure elucidation of pyridomycin analogue from $\Delta p y r 2$ mutant HTT12}

The differences in the MS/MS patterns of the peak from $\Delta p y r 2$ mutant HTT12 and wild type producer encouraged a large-scale fermentation to isolate the pyridomycin related compound.

170 Following previous reported procedures (24), a 19-L culture of the mutant HTT12 was subjected for 171 ethyl acetate extraction and subsequent column chromatography resulted in the isolation of compound HTT12-1.

HTT12-1 was isolated as a white powder. The molecular formula $\mathrm{C}_{27} \mathrm{H}_{32} \mathrm{~N}_{4} \mathrm{O}_{8}$ was established based on its HR-MS data at $m / z$ 541.2291 (calcd. 541.2293 for $[\mathrm{M}+\mathrm{H}]^{+}$), requiring 14 degrees of $(3 \mathrm{H}, \mathrm{t}, J=7.5 \mathrm{~Hz}), 1.00(3 \mathrm{H}, \mathrm{d}, J=7.0 \mathrm{~Hz}), 1.38(3 \mathrm{H}, \mathrm{d}, J=7.0 \mathrm{~Hz})$ and $1.53(3 \mathrm{H}, \mathrm{s})]$, four

$179 J=8.5,4.3 \mathrm{~Hz})$ and $7.41(1 \mathrm{H}, \mathrm{d}, J=8.5 \mathrm{~Hz})]$, a meta-disubstituted pyridine moiety $\left[\delta_{\mathrm{H}} 8.39(1 \mathrm{H}, \mathrm{dd}\right.$, $J=4.8,1.3 \mathrm{~Hz}), 8.22(1 \mathrm{H}, \mathrm{d}, J=1.3 \mathrm{~Hz}), 7.56(1 \mathrm{H}, \mathrm{d}, J=7.7 \mathrm{~Hz})$ and $7.34(1 \mathrm{H}, \mathrm{d}, J=7.7,4.8 \mathrm{~Hz})]$

181 and a series of aliphatic multiplets. The ${ }^{13} \mathrm{C}$ NMR spectrum, in combination with HSQC experiment

182 resolved 27 carbon resonances consistent with four methyls, two $s p^{3}$ methylenes, one $s p^{3}$ methine,

183 four oxygenated or nitrogenated $s p^{3}$ tertiary carbons, a tetrasubstituted double bond, two pyridine 184 units, and four ester or amide carbonyl groups. As 13 of the 14 DOUs were attributed to two pyridine 185 units, a double bond and four carbonyl groups, the remaining for DOUs required for HTT12-1 was 
monocycle. The aforementioned data were similar to the pyridomycin (1), except for the absence of

$187 \Delta^{21^{\prime}}$ in 1 and the formation of $\Delta^{1011}$ in HTT12-1 (16) (Table 1, Figure S1-6).

188 The planner structure of HTT12-1 was further established by detailed interpretation of its 2D

189 NMR spectra. Four spin systems (C-2-C-13, C-16-C-13-C-14-C-15, C-6-C-5-C-18, and C-9-C-

190 19) as depicted in Figure S6 were established based on the ${ }^{1} \mathrm{H}-{ }^{1} \mathrm{H}$ COSY spectrum. These fragments,

191 ester or amide carbonyl groups, tetrasubstituted double bond, tertiary methyl, and the oxygenated or

192 nitrogenated methines were further linked by the HMBC spectrum from $\mathrm{H}-2$ to $\mathrm{C}-12, \mathrm{H}_{3}-17$ to $\mathrm{C}$ -

193 10/C-11/C-12, H-9 to C-10/C-20, $\mathrm{H}_{2}-19$ to C-10/C-21/C-25, H-5 to C-3/C-7, H-6 to C-1'/C-7, and H-

194 4' to C-1', which generated a monocycle ring system. Especially, the key HMBC correlations from

$195 \mathrm{H}-9, \mathrm{H}_{3}-17$ and $\mathrm{H}_{2}-19$ to $\mathrm{C}-10$, and from $\mathrm{H}-2$ and $\mathrm{H}_{3}-17$ to $\mathrm{C}-12$, combined with the downfield-

196 shifted chemical shift of C-10 $\left(\delta_{\mathrm{C}} 175.4\right)$ and upfield-shifted chemical shift of C-2 $\left(\delta_{\mathrm{C}} 76.6\right)$, revealed

197 the enolic hydroxy group and 1-methylpropyl group at C-10 and C-2, respectively. Thus, the gross

198 structure of HTT12-1 was established (Figure 1). The relative configurations of HTT12-1 was

199 assigned to be the same as pyridomycin by comparing their 1D NMR data, while the relative

200 configurations of C-2 and C-13 were not determined. We therefore named compound HTT12-1 as

201 pyridomycin B (4) for the similar scaffold with pyridomycin.

\section{Determination of Pyr2 active sites}

Pyridomycin B featured with enol moiety in pyridyl alanine moiety and a saturated 3-

methylvaleric acid group. Inspired by the chemical structure of pyridomycin B, we reexamined the

role of Pyr2. The classical SDRs family is known for sharing the canonical NAD(P)-binding motif,

and this glycine-rich region plays a critical role in domain stability as well as allows access of the

207 NAD(P) pyrophosphate. Comparison of the primary sequences of Pyr2 and its homologues revealed

208 TGGxxGxG, the highly conserved Rossmann fold of classical SDR subfamily. In most representative 

acts as a catalytic acid/base which forms a hydroxyl-tyrosinate ion that donates or abstracts a proton

211 from the substrate (26). Intriguingly, alignment of $\mathrm{Pyr} 2$ with other canonical SDR family

212 oxidoreductases reveals that Pyr2 contains a His instead of the usually conserved Tyr such that the

213 active site triad is S140-H154-K158 (Figure 2).

214 Since the active triad S-H-K in Pyr2 is different from the conserved catalytic triad of typical

215 SDR family proteins, we performed site-directed mutagenesis to probe the functions of the three key

216 residues. Encouraged by the successful complementation of mutant HTT12 with Pyr2, we mutated

217 these residues to Ala respectively using pJTU4659 as template to test in vivo if these Pyr2 variants

218 retained the ability of pyridomycin B production. The resulting plasmids containing pyr2 with point

219 mutation were introduced into HTT12 mutant by conjugation for complementation. Pyridomycin and

220 pyridomycin B showed some different MS/MS fragmentation patterns, we therefore chose extracted

221 ion chromatograms (EIC) of fragment $m / z 411$ and $m / z 409$, respectively for comparison. After

222 carefully analyzing the MS/MS profile, we found that mutating of the His154 residue to Alanine

223 (Pyr2/H154A) cannot restore the production of pyridomycin. Both of the Pyr2/S140A and

224 Pyr2/K158A mutant exhibit the fragment $m / z$ 411, which means the complementation of pyridomycin

225 production. Moreover, we mutated the His154 to Tyr and identified that Pyr2/H154Y mutant endow

226 the capability of pyridomycin production (Figure 4). Such observations suggest that the catalytic triad 227 engages in a different manner and His154 is critical for the function of Pyr2.

\section{Discussion}

230 Tuberculosis (TB) is one of the world's leading causes of death. Since the discovery of pyridomycin 231 as the antituberculous natural product over 60 years ago, it hasn't garnered considerable attention 
232 until being demonstrated as a target inhibitor of InhA enoyl reductase of Mtb (28). Recently,

233 significant progress in understanding the antituberculous mechanism inspired generation of a serious

234 pyridomycin derivatives for SAR studies $(13-15,24)$. Generating pyridomycin analogues by genetic

235 manipulation is an alternative approach based upon understanding of the biosynthetic mechanisms. In

236 this work, we report the characterization of a new pyridomycin analogue from a pyr2 knockout

237 mutant HTT12, which lays a foundation for utilization of the pyridomycin pathway to develop

238 promising antituberculous derivatives by genetic engineering.

Pyr2 is a 3-ketoacyl-ACP reductase, which belongs to the SDR superfamily. The draft genome

sequence analysis of S. pyridomyceticus revealed the presence of additional three Pyr2 homologues

241 (Figure S7) with $>90 \%$ coverage of varying homology (>30\% identities, $\sim 52 \%$ similarities).

242 Although the overall structure of Pyr2 and SDR proteins are highly analogous, the catalytic

243 conformation of Pyr2 may diverge from other classical SDR family oxidoreductases as the variation

244 of the active site triad. In addition, $\Delta p y r 2$ mutant HTT12 lost the productivity of pyridomycin,

245 showing that these homologues could not restore the function of Pyr2. It clearly indicates that Pyr2 is

246 pathway specific for pyridomycin biosynthesis.

247 Previous biosynthetic analysis revealed that among the NRPS-PKS assembly line of pyridomycin,

248 PKS module PyrF (KS-AT-MT-ACP) incorporates the propionic acid derived unit. According to the

249 chemical structure of final product, there is a requirement for the reduction of $\mathrm{C}-10$ keto of the linear

250 peptide-polyketide intermediate. Notably, the PKS PyrF lacks a KR domain for hydroxyl group

251 formation, in contradiction to the presence of hydroxyl group (*) at C-10 of pyridomycin. Several

252 examples of standalone ketoreductase from assembly-line system have been reported. For example,

253 AntM perfroms a ketoreduction of the C-8 carbonyl of the linear ACP-bound intermediate in

254 antimycin pathway (22). SimC7 is a ketoreductase that reduces the carbonyl to a hydroxyl group at

255 the C-7 position in simocyclione D8 (23). It may hint that the Pyr2 contributes to the formation of the 
C-10 hydroxyl group. In vivo knockout of pyr2 accumulated a new pyridomycin analogue,

pyridomycin B. Although pyridomycin B specifically featured the enoyl group at C-10-C-11, we speculated that the enol form presented as the major species in equilibrium instead of the keto form due to the bulky aryl group of pyridomycin B at C-9 and dicarbonyl at C-10 and C-12. Isolation of pyridomycin B implies that Pyr2 functions as a trans ketoreductase, acts upon an acyl carrier proteinbound linear biosynthetic intermediate and catalyzes the formation of hydroxyl group on C-10 (*) prior to TE-catalyzed cyclization. We also purified Pyr2 protein and carried out in vitro experiment using pyridomycin B as the substrate. Unfortunately, no detectable new product in the reaction indicated that Pyr2 catalyzes a reaction on ACP or PCP-tethered substrate (data not show).

The enolic acid moiety of pyridomycin is essential for antibiotic activity, interacting with the substrate binding pocket and resulting in competitive inhibition (29). Synthesis of the enol moiety is also an obstacle in de novo synthesis of pyridomycin. Pyridomycin B harbors a saturated 3methylvaleric acid group rather than an enol moiety. Based on the structure of pyridomycin B, along with the fact that there is no other dehydrogenase within this gene cluster, it seems that Pyr2 is also responsible for the double bond $(\mathrm{C}-2, \#)$ formation in the isoleucine-derived moiety. We then proposed that the NAD(P) dependent enzyme Pyr2 may act as a bifunctional enzyme that abstracts chain for $\mathrm{NAD}(\mathrm{P}) \mathrm{H}$ formation, and then uses the NADPH formed in situ to reduce the $\beta$-keto $(*)$ to generate a hydroxyl group (Figure 5), suggesting an atom economy manner. biosynthesis. The varied catalytic triad of Pyr2 suggests that the function of Pyr2 is different from the 
strategy. The enoic acid moiety is known to be an important pharmacophoric group in pyridomycin.

281 Studies in future including functional characterization and structural elucidation of Pyr2 will lead to

282 defining the essential motif and major active-residues of Pyr2 for interacting with the substrate,

283 which would help to introduce other enol carboxylic acids to expand structural diversity of

284 pyridomycin by engineering Pyr2 for antimycobacterial activity screening.

\section{Materials and Methods}

\section{Bacterial strains, plasmids, and culture conditions}

287 Bacterial strains, plasmids and oligo primers for PCR amplification used in this study are 288 summarized in Table 2-3. PCRs were performed using rTaq (Takara biotechnology, Dalian, China)

289 or KOD (Toyobo, Japan), and the PCR primers were synthesized by Invitrogen (Shanghai, China).

290 Overlapping-PCR based site-directed mutagenesis was employed to introducing point mutations of

291 pyr2. Restriction endonucleases were purchased from Thermo Fisher and the reactions were

292 performed according to the manufacture's procedures. Other common biochemical, chemical

293 solvents and media components were purchased from standard commercial sources and used directly.

294 E. coli strains were routinely grown in LB medium at $37^{\circ} \mathrm{C}$ and supplemented with appropriate

295 antibiotics for plasmid maintenance. Streptomyces pyridomyceticus and the derived mutants were

296 cultured in liquid tryptic soy broth (TSB) for mycelium, on solid COM medium for sporulation, on

297 2CM medium for conjugation and in fermentation medium (2.5\% glucose, $1.5 \%$ soybean meal, $0.5 \%$

$298 \mathrm{NaCl}, 0.05 \% \mathrm{KCl}, 0.025 \% \quad \mathrm{MgSO}_{4} \cdot 7 \mathrm{H}_{2} \mathrm{O}, 0.3 \% \quad \mathrm{~K}_{2} \mathrm{HPO}_{4}, 0.3 \% \quad \mathrm{Na}_{2} \mathrm{HPO}_{4} \cdot 12 \mathrm{H}_{2} \mathrm{O}, \mathrm{pH}$ 7.2) for

299 production of pyridomycin and pyridomycin B. E. coli-Streptomyces conjugations were performed 300 following previously reported protocols (30).

\section{DNA sequencing and bioinformatics analysis}


302 The draft genome was submitted to secondary metabolite gene cluster analysis using antiSMASH

303 (31). A PSI-BLAST search of the NCBI nonredundant homology search was conducted on the pyr2

304 and a tblastn search performed by BioEdit. The NRPS and PKS architectures were predicted by

305 NRPS-PKS predictor (http://nrps.igs.umaryland.edu/) and confirmed against GenBank database by

306 BLAST by manual inspection. Multiple sequence alignments and local blast analysis were performed

307 using BioEdit Sequence Alignment Editor. The Pyr2 sequence was aligned with ClustalW using

308 BioEdit and phylogenetic analyses were performed by MEGA-X with 1000 bootstrap replications

309 (32). Using Pyr2 as the query, we searched for homologous proteins in the nonredundant database of

310 Genbank and create the sequence similarity networks (SNN) using Enzyme Function Initiative (EFI)

311 tools $(33,34)$. Cytoscape V6.0 was used for the SNN visualization and analysis.

\section{Complementation of $\Delta p y r 2$ mutant HTT12}

313 In pyr2 inactivation mutant $S$. pyridomyceticus HTT12, the pyr2 was replaced by kanamycin

314 resistance cassette using a standard $\lambda$ Red-mediated PCR targeting recombination system. For the

315 construction of the gene complementation construct, pyr2 was amplified from S. pyridomyceticus

316 genomic DNA by PCR using high-fidelity DNA polymerase and digest with NdeI-EcoRI. The

317 resulting fragment was cloned downstream of the PermE* promoter into pIB139, the E. coli-

318 Streptomyces expression shuttle vector, digested with the corresponding restriction enzymes to yield

319 the complementary plasmid pJTU4656 (Table S1). To achieve the point mutations in Pyr2, 320 pJTU4656 (H154Y), pJTU4656 (H154A), pJTU4656 (S154Y), and pJTU4656 (K154Y) were

321 constructed by site-directed mutagenesis using the primers listed in Table 2. The plasmid pJTU4656

322 and its derivatives carrying point mutations in pyr 2 were introduced into mutant HTT12 respectively

323 by intergeneric conjugation as previously described. The resultant mutant strains were selected for 324 using kanamycin resistance. 
325 Fermentation, production, and HPLC analysis of pyridomycin and derivatives

326 The $\Delta p y r 2$ mutant HTT12 were cultured following previously reported procedures (24), with the wild

327 type producer as a control. The seed culture was prepared by inoculating $25 \mathrm{~mL}$ of TSB medium in

$328250 \mathrm{~mL}$ flask with fresh spore from COM agar plate and incubating at $30^{\circ} \mathrm{C}$ for $24 \mathrm{~h}$ on a rotary

329 shaker $(220 \mathrm{rpm})$. The seed culture was inoculated into a fermentation medium at 5\%, and continued

330 to incubate for 3 days. The fermentation broth was extracted three times with ethyl acetate, and the

331 combined extract was concentrated in a vacuum evaporator to generate a solid residue. The resulting

332 residue was dissolved in $1 \mathrm{~mL}$ methanol, centrifuged and filtered prior to analysis. HPLC-MS

333 analysis was performed on a ZORBAX RX-C18 column (150×4.6 mm, $5 \mu \mathrm{m}$, Agilent). The column

334 was first equilibrated with $80 \%$ solvent A (0.1\% formic acid in water) and $20 \%$ solvent B $(0.1 \%$

335 formic acid in acetonitrile). After 5 min of isocratic flow, a linear gradient was developed with a 25-

336 min linear gradient from $20 \% \mathrm{~B}$ to $80 \% \mathrm{~B}$ at a flow rate of $0.5 \mathrm{~mL} / \mathrm{min}$ and $\mathrm{UV}$ detection at $305 \mathrm{~nm}$.

337 The mass spectrometer was run in positive ion detection mode and set to scan between 100 and 800 $338 \mathrm{~m} / \mathrm{z}$.

\section{Isolation and structure elucidation of pyridomycin B}

340 Large-scale fermentation (19 L) was carried out to isolate sufficient quantities of metabolite from the

341 spyr2 mutant HTT12 for structural elucidation. The supernatant was separated from mycelia by

342 centrifugation (8000 rpm, $15 \mathrm{~min}$ ), and was extracted three times with $10 \mathrm{~L}$ of ethyl acetate (EtOAc).

343 The organic solvent in the combined extracts were removed under reduced pressure. The resulted

344 crude extract (4.28 g) was adsorbed to $200 \mathrm{~g} \mathrm{RP}-18$ reverse phase resin (YMC group, Japan) and was

345 eluted with a gradient of $\mathrm{CH}_{3} \mathrm{OH}-\mathrm{H}_{2} \mathrm{O}(20: 80,30: 70,50: 50,70: 30$ and 100:0) at $3 \mathrm{~mL} / \mathrm{min}$ to give

346120 fractions. Each fraction was tested for alkaloid compounds by bismuth potassium iodide solution.

347 Fractions with positive signals (fraction 69-83) were combined and concentrated to give $120 \mathrm{mg}$ Fr2, 
348 further purified by a Sephadex LH-20 column using $\mathrm{MeOH}$ as the mobile phase at $1 \mathrm{~mL} / \mathrm{min}$ to give

349 a subfraction Fr2A (15 mg) with positive signals by TLC and UV detection. Fraction Fr2A was

350 purified by silica gel column chromatography using gradient of $\mathrm{H}_{2} \mathrm{O} / \mathrm{CH}_{3} \mathrm{OH}(70: 30,40: 60)$ at 2

$351 \mathrm{~mL} / \mathrm{min}$ to yield Fr2A1 $25 \mathrm{mg}$, and then subjected to semipreparative preparative HPLC (ZORBAX

352 Eclipse $\mathrm{XDB}-\mathrm{C} 18,9.4 \times 250 \mathrm{~mm}, 5 \mu \mathrm{m}$, Agilent) using $0.1 \%$ formic acid in water/ $\mathrm{CH}_{3} \mathrm{OH}$ as the

353 mobile phase to afford pyridomycin B (25 mg).

NMR spectra were recorded on a Bruker AV-III 600 (500 MHz) spectrometer. Chemical shifts are reported in parts per million (ppm) and coupling constants $J$ are given in Hertz $(\mathrm{Hz})$. Structural

356 elucidation of the isolated compounds was achieved by means of $1 \mathrm{D}\left({ }^{1} \mathrm{H}\right.$ and $\left.{ }^{13} \mathrm{C}\right)$ and $2 \mathrm{D}\left({ }^{1} \mathrm{H}-{ }^{1} \mathrm{H}\right.$

357 COSY, HSQC, and HMBC) NMR spectra (Figure S1-S6) and by comparison of the spectra with 358 published data (16). The NMR data are summarized in Table 3. Samples were dissolved in $\mathrm{CD}_{3} \mathrm{OD}$.

359 High resolution MS analysis was performed on an Agilent 1200 series LC/MSD trap system in 360 tandem with a 6530 Accurate-Mass Q-TOF mass spectrometer with an ESI source $(50-800 \mathrm{~m} / \mathrm{z}$ mass 361 range at positive mode).

\section{Antimicrobial activity assay}

363 The antimycobacterial activities of the pyridomycin producers against indicator strain

364 Mycobacterium smegmatis mc $^{2} 155$ using a standard diffusion assay. The $20 \mu \mathrm{L}$ crude extracts of $S$.

365 pyridomyceticus strains were dropped on paper disks and placed on the solid LB agar plates

366 containing $\sim 10^{7}$ M. smegmatis $\mathrm{mc}^{2} 155$ cell. The plates were incubated overnight at $37^{\circ} \mathrm{C}$ and halo

367 zone of inhibition were observed. Pyridomycin was used a positive control.

\section{SUPPLEMENTAL MATERIAL}

369 Supplemental File including supplemental figure 1-7. 


\section{ACKNOWLEDGMENTS}

This work was supported by the grants from National Natural Science Foundation of China

372 (31970053 and 32170059 to T.H., 21632007 to S.L), the National Key Research and Development

373 Program of China (2018YFA0901900), and the Startup fund for Youngman Research at SJTU

374 (SFYR at SJTU), respectively. We would like to thank the Instrumental Analysis Center of Shanghai

375 Jiao Tong University and Shanghai Institute of Organic Chemistry for obtaining the NMR data.

\section{References}

378

379

380

381

382

383

384

385

386

387

388

389

390

391

392

393

394

395

396

397

398

399

400

401
1. WHO. 2018. 2018 Global tuberculosis report. World Health Organization Press W, Geneva, Switzerland

2. Lechartier B, Rybniker J, Zumla A, Cole ST. 2014. Tuberculosis drug discovery in the postpost-genomic era. EMBO Mol Med 6:158-68.

3. Dong M, Pfeiffer B, Altmann KH. 2017. Recent developments in natural product-based drug discovery for tuberculosis. Drug Discovery Today 22:585-591.

4. Herrmann J, Rybniker J, Muller R. 2017. Novel and revisited approaches in antituberculosis drug discovery. Curr Opin Biotechnol 48:94-101.

5. Baptista R, Bhowmick S, Nash RJ, Baillie L, Mur LA. 2018. Target discovery focused approaches to overcome bottlenecks in the exploitation of antimycobacterial natural products. Future Med Chem 10:811-822.

6. Riccardi G, Pasca MR. 2014. Trends in discovery of new drugs for tuberculosis therapy. J Antibiot (Tokyo) 67:655-659.

7. Pan P, Tonge PJ. 2012. Targeting InhA, the FASII Enoyl-ACP Reductase: SAR Studies on Novel Inhibitor Scaffolds. Current Topics in Medicinal Chemistry 12:672-693.

8. Rozman K, Sosic I, Fernandez R, Young RJ, Mendoza A, Gobec S, Encinas L. 2017. A new 'golden age' for the antitubercular target inha. Drug Discovery Today 22:492-502.

9. Vilcheze C, Jacobs WR, Jr. 2007. The mechanism of isoniazid killing: clarity through the scope of genetics. Annu Rev Microbiol 61:35-50.

10. Hartkoorn RC, Sala C, Neres J, Pojer F, Magnet S, Mukherjee R, Uplekar S, Boy-Rottger S, Altmann KH, Cole ST. 2012. Towards a new tuberculosis drug: pyridomycin - nature's isoniazid. Embo Molecular Medicine 4:1032-1042.

11. Maeda K, Kosaka H, Okami Y, Umezawa H. 1953. A new antibiotic, pyridomycin. J Antibiot (Tokyo) 6:140. 
402

403

404

405

406

407

408

409

410

411

412

413

414

415

416

417

418

419

420

421

422

423

424

425

426

427

428

429

430

431

432

433

434

435

436

437

438

439

440

441

12. Ogawara H, Maeda K, Umezawa H. 1968. The biosynthesis of pyridomycin. I. Biochemistry 7:3296-3302.

13. Hartkoorn RC, Pojer F, Read JA, Gingell H, Neres J, Horlacher OP, Altmann KH, Cole ST. 2014. Pyridomycin bridges the NADH- and substratebinding pockets of the enoyl reductase InhA. Nature Chemical Biology 10:96-98.

14. Horlacher OP, Hartkoorn RC, Cole ST, Altmann KH. 2013. Synthesis and Antimycobacterial Activity of 2,1 '-Dihydropyridomycins. Acs Medicinal Chemistry Letters 4:264-268.

15. Kienle M, Eisenring P, Stoessel B, Horlacher OP, Hasler S, van Colen G, Hartkoorn RC, Vocat A, Cole ST, Altmann KH. 2020. Synthesis and Structure-Activity Relationship Studies of C2-Modified Analogs of the Antimycobacterial Natural Product Pyridomycin. J Med Chem 63:1105-1131.

16. Kinoshita M, Nakata M, Takarada K, Tatsuta K. 1989. Synthetic Studies of Pyridomycin .5. Total Synthesis of Pyridomycin. Tetrahedron Letters 30:7419-7422.

17. Wash CT, Tang Y. 2017. Natural Product Biosynthesis: Chemical Logic and Enzymatic Machinery. Royal Society of Chemistry, CPI Group (UK) Ltd, Croydon, CR04YY, UK.

18. Piel J. 2010. Biosynthesis of polyketides by trans-AT polyketide synthases. Natural Product Reports 27:996-1047.

19. Helfrich EJN, Piel J. 2016. Biosynthesis of polyketides by trans-AT polyketide synthases. Natural Product Reports 33:231-316.

20. Heneghan MN, Yakasai AA, Williams K, Kadir KA, Wasil Z, Bakeer W, Fisch KM, Bailey AM, Simpson TJ, Cox RJ, Lazarus CM. 2011. The programming role of trans-acting enoyl reductases during the biosynthesis of highly reduced fungal polyketides. Chemical Science 2:972-979.

21. Zou Y, Yin H, Kong D, Deng Z, Lin S. 2013. A trans-acting ketoreductase in biosynthesis of a symmetric polyketide dimer SIA7248. Chembiochem 14:679-683.

22. Fazal A, Hemsworth GR, Webb ME, Seipke RF. 2021. A Standalone beta-Ketoreductase Acts Concomitantly with Biosynthesis of the Antimycin Scaffold. ACS Chem Biol 16:1152-1158.

23. Schafer M, Stevenson CEM, Wilkinson B, Lawson DM, Buttner MJ. 2016. SubstrateAssisted Catalysis in Polyketide Reduction Proceeds via a Phenolate Intermediate. Cell Chem Biol 23:1091-1097.

24. Huang TT, Wang YM, Yin J, Du YH, Tao MF, Xu J, Chen WQ, Lin SJ, Deng ZX. 2011. Identification and Characterization of the Pyridomycin Biosynthetic Gene Cluster of Streptomyces pyridomyceticus NRRL B-2517. Journal of Biological Chemistry 286:2064820657.

25. Huang TT, Li LL, Brock NL, Deng ZX, Lin SJ. 2016. Functional Characterization of PyrG, an Unusual Nonribosomal Peptide Synthetase Module from the Pyridomycin Biosynthetic Pathway. Chembiochem 17:1421-1425.

26. Kavanagh K, Jornvall H, Persson B, Oppermann U. 2008. The SDR superfamily: functional and structural diversity within a family of metabolic and regulatory enzymes. Cellular and Molecular Life Sciences 65:3895-3906. 
27. Filling C, Berndt KD, Benach J, Knapp S, Prozorovski T, Nordling E, Ladenstein R, Jornvall H, Oppermann U. 2002. Critical residues for structure and catalysis in short-chain dehydrogenases/reductases. J Biol Chem 277:25677-25684.

28. Wright GD. 2012. Back to the future: a new 'old' lead for tuberculosis. Embo Molecular Medicine 4:1029-1031.

29. Hartkoorn RC, Pojer F, Read JA, Gingell H, Neres J, Horlacher OP, Altmann KH, Cole ST. 2014. Pyridomycin bridges the NADH- and substrate-binding pockets of the enoyl reductase InhA. Nat Chem Biol 10:96-98.

30. Kieser T, Bibb, M. J., Chater, K. F., Butter, M. J., and Hopwood, D. 2000. Practical Streptomyces Genetics: A Laboratory Manual. John Innes Foundation, Norwich, UK.

31. Blin K, Shaw S, Steinke K, Villebro R, Ziemert N, Lee SY, Medema MH, Weber T. 2019. antiSMASH 5.0: updates to the secondary metabolite genome mining pipeline. Nucleic Acids Research 47:W81-W87.

32. Kumar S, Stecher G, Li M, Knyaz C, Tamura K. 2018. MEGA X: Molecular Evolutionary Genetics Analysis across Computing Platforms. Mol Biol Evol 35:1547-1549.

33. Gerlt JA. 2017. Genomic Enzymology: Web Tools for Leveraging Protein Family SequenceFunction Space and Genome Context to Discover Novel Functions. Biochemistry 56:42934308. 
465 TABLE $1{ }^{1} \mathrm{H}(500 \mathrm{MHz})$ and ${ }^{13} \mathrm{C}(125 \mathrm{MHz})$ NMR data of pyridomycin $\mathbf{B}(\delta$ in ppm).

\begin{tabular}{|c|c|c|c|c|c|}
\hline position & $\delta_{\mathrm{H}}^{b}$, multi $(J$ in $\mathrm{Hz})$ & $\delta_{\mathrm{C}}$, type & position & $\delta_{\mathrm{H}}^{b}$, multi $(J$ in $\mathrm{Hz})$ & $\delta_{\mathrm{C}}$, type \\
\hline 2 & $4.06, \mathrm{~d}(4.5)$ & $76.6, \mathrm{CH}$ & 18 & $1.38, \mathrm{~d}(7.0)$ & $18.5, \mathrm{CH}_{3}$ \\
\hline \multirow[t]{2}{*}{3} & & $175.2^{c}, \mathrm{C}$ & 19 & $3.58, \mathrm{dd}(14.0,4.0)$ & $32.7, \mathrm{CH}_{2}$ \\
\hline & & & & $3.18, \mathrm{dd}(14.0,2.5)$ & \\
\hline 5 & $5.57, \mathrm{~m}$ & $71.5, \mathrm{CH}$ & 20 & & 133.1, C \\
\hline 6 & $5.88, \mathrm{~m}$ & $57.3, \mathrm{CH}$ & 21 & 7.56, brd $(7.5)$ & 141.1, CH \\
\hline 7 & & $169.8, \mathrm{C}$ & 22 & $7.34, \mathrm{dd}(7.5,5.0)$ & $125.4, \mathrm{CH}$ \\
\hline 9 & $4.61, \mathrm{brs}$ & $60.5, \mathrm{CH}$ & 23 & $8.39, \mathrm{dd}(5.0,1.0)$ & $148.0, \mathrm{CH}$ \\
\hline 10 & & $175.4^{c}, \mathrm{C}$ & 25 & $8.22, \mathrm{~d}(1.5)$ & $150.2, \mathrm{CH}$ \\
\hline 11 & & 103.4, C & $1^{\prime}$ & & $170.9, \mathrm{C}$ \\
\hline 12 & & 174.3, C & $2^{\prime}$ & & 132.5, C \\
\hline 13 & $1.86, \mathrm{~m}$ & $40.4, \mathrm{CH}$ & $3^{\prime}$ & & 159.5, C \\
\hline 14 & $1.54, \mathrm{~m}$ & $25.2, \mathrm{CH}_{2}$ & $4^{\prime}$ & 7.41, brd $(8.5)$ & 127.7, $\mathrm{CH}$ \\
\hline 15 & $0.96, \mathrm{t}(7.5)$ & $12.4, \mathrm{CH}_{3}$ & $5^{\prime}$ & $7.51, \mathrm{dd}(8.5,4.0)$ & $130.8, \mathrm{CH}$ \\
\hline 16 & $1.00, \mathrm{~d}(7.0)$ & $16.0, \mathrm{CH}_{3}$ & $6^{\prime}$ & $8.19, \mathrm{~d}(4.0,1.0)$ & $141.5, \mathrm{CH}$ \\
\hline 17 & $1.53, \mathrm{~s}$ & $5.7, \mathrm{CH}_{3}$ & & & \\
\hline
\end{tabular}

$466{ }^{a}$. in $\mathrm{CD}_{3} \mathrm{OD} .^{b}$. "m" means overlapped or multipet with other signals. ${ }^{c}$. may be interchanged in each 467 column. 
TABLE 2 Plasmids and posmids used in this study.

\begin{tabular}{|c|c|c|}
\hline Strains & Relevant phenotype & $\begin{array}{c}\text { Source/ } \\
\text { References }\end{array}$ \\
\hline \multicolumn{3}{|c|}{ Streptomyces pyridomyceticus } \\
\hline NRRL B-2517 & Wild type producer of pyridomycin & $\begin{array}{l}\text { ARS Culture } \\
\text { Collection }\end{array}$ \\
\hline HTT12 & $\begin{array}{l}\text { S. pyridomyceticus mutant with pyr } 2 \text { gene deletion by PCR targeting strategy, } \\
\text { kanamycin resistance }\end{array}$ & \\
\hline HTT12::pJTU4656 & $\begin{array}{l}\text { HTT5 complemented with pJTU4656 harboring full length of pyr2, apramycin } \\
\text { and kanamycin resistance }\end{array}$ & This work \\
\hline \multicolumn{3}{|l|}{ Escherichia coli } \\
\hline$\overline{\mathrm{DH} 10 \mathrm{~B}}$ & $\begin{array}{l}\text { E. coli host for general cloning }\left[\mathrm{F}^{-} \text {mcrA } \Delta(\text { mrr- } h s d R M S \text { - mcrBC }) \varphi 80 \text { dlacZ }\right. \\
\Delta M 15 \Delta \text { lacX74 deoR recAl endAl ara } \Delta 139 \mathrm{D} \text { (ara,leu }) 1697 \text { galU galK } \lambda^{-} r s p L \\
\text { nupG] }\end{array}$ & \\
\hline ET12567/pUZ8002 & $\begin{array}{l}\text { Methylation-deficient } E \text {. coli host for intergeneric conjugation }[\mathrm{rec} F, d a m, d c m \text {, } \\
\left.h s d \mathrm{~S}, \mathrm{Cml}^{\mathrm{R}}, \mathrm{Km}^{\mathrm{R}}\right]\end{array}$ & \\
\hline BW25113/pIJ790 & E. coli host for $\lambda$ RED-mediated PCR targeting & \\
\hline \multicolumn{3}{|l|}{ Plasmids } \\
\hline pIB139 & $\operatorname{aac}(3) I V$, int, attФC31, reppuc,oriT, PermE & \\
\hline Cosmid 3C10 & $\begin{array}{l}\text { An end-sequenced cosmid which contains partial pyridomycin biosynthetic gene } \\
\text { cluster }\end{array}$ & This study \\
\hline 3C10pyr2-kan & $\begin{array}{l}\text { The pyr2 in cosmid 13C6 substituted using the PCR-targeting method by } \\
\text { kanamycin cassette which amplified with primers Pyr } 2 \text { tgtF and Pyr2tgtR }\end{array}$ & (24) \\
\hline pJTU4656 & $\begin{array}{l}\text { The PCR amplified pyr2 from cosmid } 3 \mathrm{C} 10 \text { was cloned into the NdeI and EcoRI } \\
\text { site of pIB139 }\end{array}$ & $(24)$ \\
\hline pJTU4656-H154Y & $\begin{array}{l}\text { Site-directed mutagenesis of histidine(154) to tyrosine in pyr2 by overlapping } \\
\text { PCR using pJTU4656 as template }\end{array}$ & This study \\
\hline pJTU4656-H154A & $\begin{array}{l}\text { Site-directed mutagenesis of histidine(154) to alanine in } p y r 2 \text { by overlapping } \\
\text { PCR using pJTU4656 as template }\end{array}$ & This study \\
\hline pJTU4656-S140A & $\begin{array}{l}\text { Site-directed mutagenesis of serine (140) to alanine in } p y r 2 \text { by overlapping PCR } \\
\text { using pJTU } 4656 \text { as template }\end{array}$ & This study \\
\hline pJTU4656-K158A & $\begin{array}{l}\text { Site-directed mutagenesis of lysine (158) to alanine in } p y r 2 \text { by overlapping PCR } \\
\text { using pJTU } 4656 \text { as template }\end{array}$ & This study \\
\hline
\end{tabular}

471 TABLE 3 Primers used in this study

\begin{tabular}{ll}
\hline Primers & Sequence \\
\hline Pyr2tgtF & ATGACTCTTTCGCAGAAGAAGGCGCTGGTCACCGGAGGCATTCCGGGGATCCGTCGACC \\
Pyr2tgtR & TCAGACCATGCCGCCGGTCACGCGCAGGTTCTGGCCGCTTGTAGGCTGGAGCTGCTTC \\
Pyr1\&2IDF & GTGCCGACCGACCGCAACCG \\
Pyr1\&2IDR & TCAGACCATGCCGCCGGTCAC \\
Pyr2expNF & GGCAATTCCATATGACTCTTTCGCAGAAGAA \\
Pyr2expER & GGAATTCTCAGACCATGCCGCCGGTC \\
Pyr2-H154Y-F & GTGTCGCTGTACGCCGGCAGCAAGGCG \\
Pyr2-H154Y-R & GCTGCCGGCGTACAGCGACACGCCGGG \\
Pyr2-H154A-F & GTGTCGCTGGCCGCCGGCAGCAAGGCG \\
Pyr2-H154A-R & GCTGCCGGCGGCCAGCGACACGCCGGG \\
Pyr2-S140A-F & ATCAACCTCGCGACCGCCAACACCGTG \\
Pyr2-S140A-R & GTTGGCGGTCGCGAGGTTGATGATGCG \\
Pyr2-K158A-F & GCCGGCAGCGCGGCGGCCGTCGAGCAG \\
Pyr2-K158A-R & GACGGCCGCCGCGCTGCCGGCGTGCAG \\
\hline
\end{tabular}


A

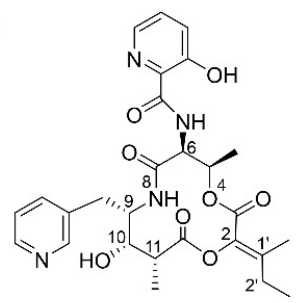

1

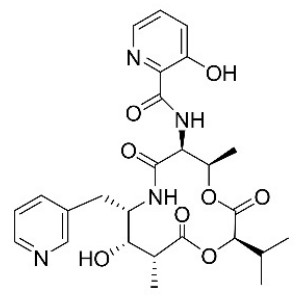

2

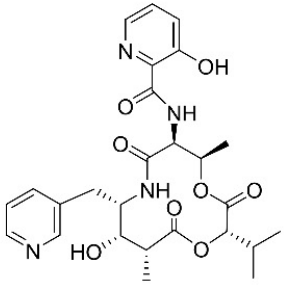

3

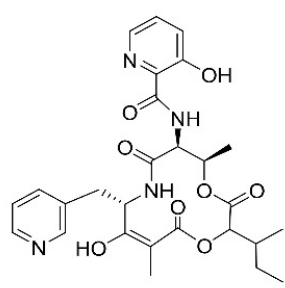

4

B
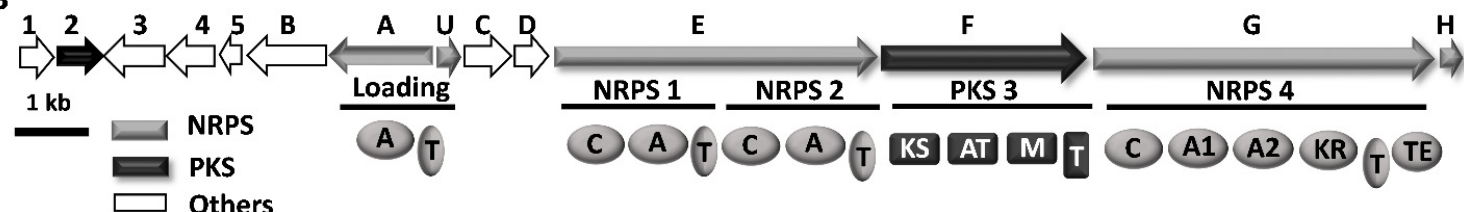

2 Figure 1. In vivo characterization of $p y r 2$ in pyridomycin biosynthesis. A. Structures of

3 pyridomycin and pyridomycin B; B. Genetic organization of the pyridomycin biosynthetic gene cluster

4 from S. pyridomyceticus B2517. Pyr2 is highlighted in black. 
bioRxiv preprint doi: https://doi.org/10.1101/2021.10.14.464479; this version posted October 15, 2021. The copyright holder for this preprint (which was not certified by peer review) is the author/funder. All rights reserved. No reuse allowed without permission.

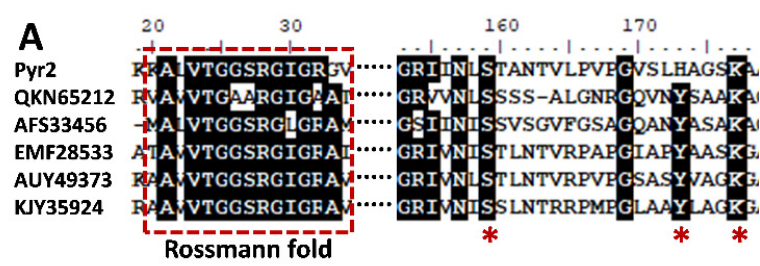

B

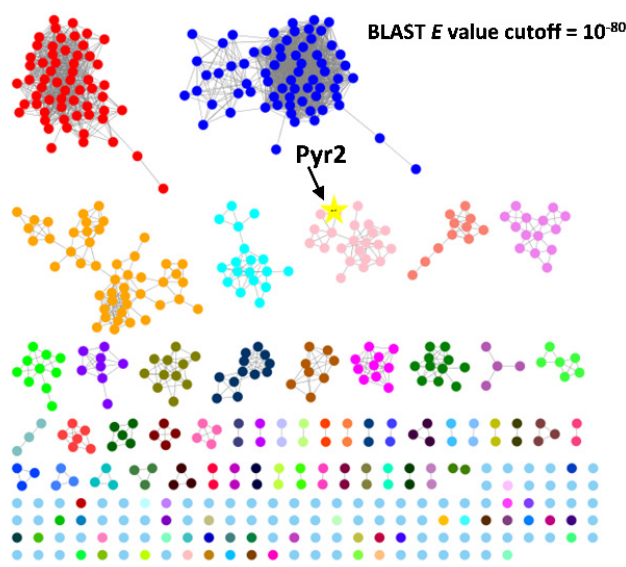

9 Figure 2. Bioinformatics analysis of Pyr2. A. Sequence alignment of selected Pyr2 homologs. The

10 rossmann fold is highlighted in dash. The catalytic residues are shown with asterisks (*). B. A sequence

11 similarity network (SSN) of Pyr2 homologs (sequence identity of $>40 \%$ ) visualized by Cytoscape. 

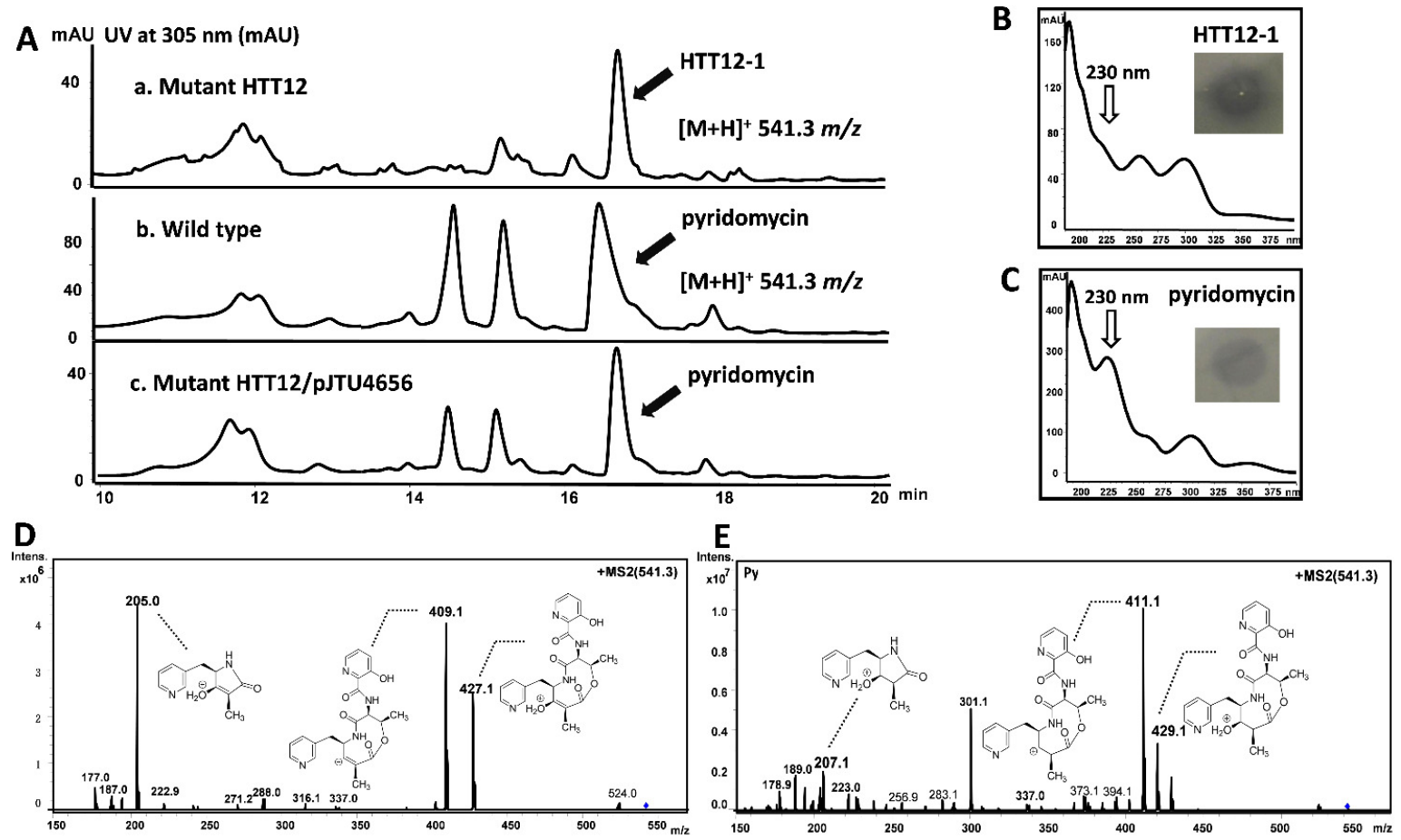

14 Figure 3. In vivo characterization of $p y r 2$ in pyridomycin biosynthesis. A. UV at $305 \mathrm{~nm}$ from

15 HPLC analysis of metabolites from wild type $S$. pyridomyceticus B2517, HTT12 (Apyr2) and

16 HTT12::pyr2. B. Bioassay against M. smegmatis mc $^{2} 155$ and UV spectrum of the peak from HTT12,

17 pyridomycin as a control (C); MS/MS spectrum of the peak from HTT12 (D) and pyridomycin (E),

18 respectively. 


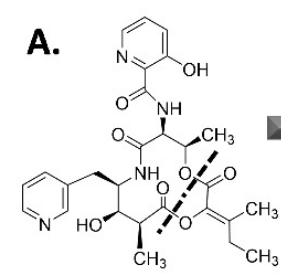

1, $[\mathrm{M}+\mathrm{H}]^{+}: \mathbf{5 4 1}$

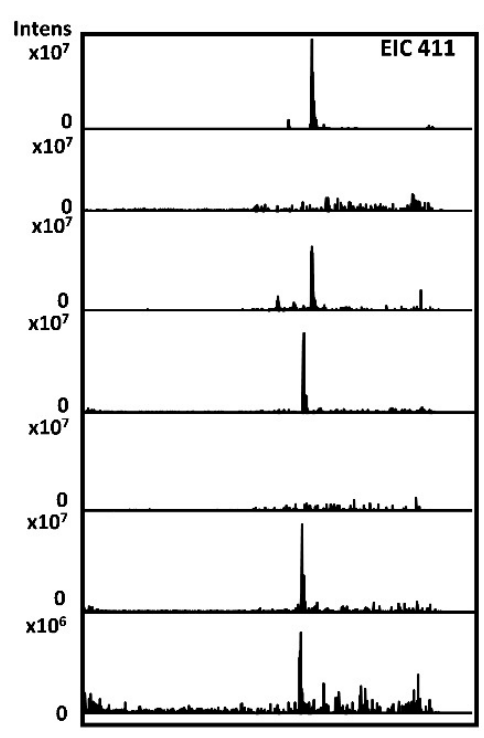

B.

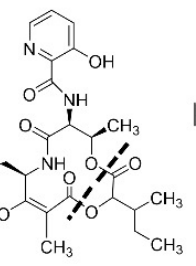

4, $[\mathrm{M}+\mathrm{H}]^{+}: 541$

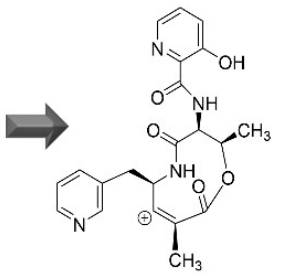

$[\mathrm{M}+\mathrm{H}]^{+}: 409$

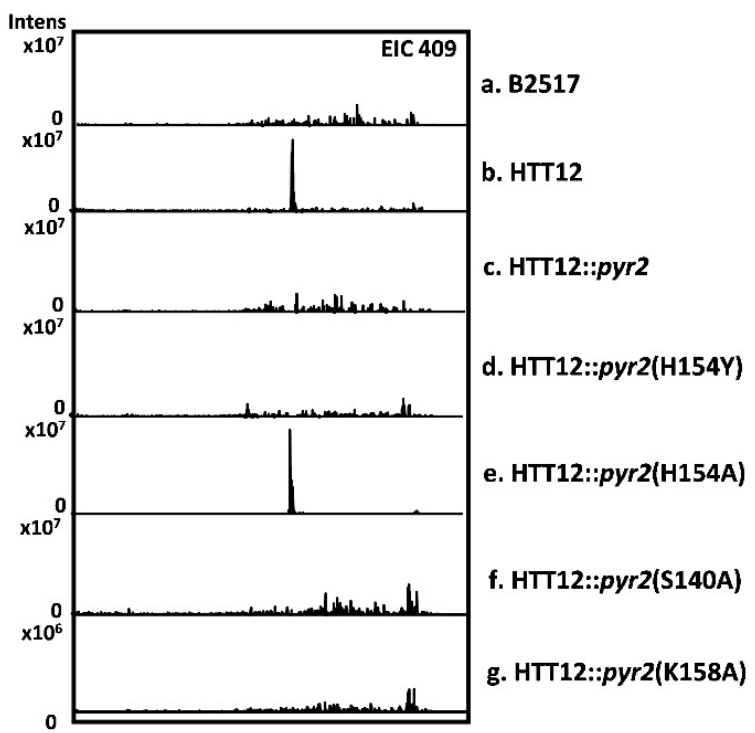

20 Figure 4. LC-MS analysis of $\boldsymbol{S}$. pyridomyceticus recombinant strains. Extracted ion chromatograms

21 (EIC, $m / z$ at 409 and 411) from LC-MS analysis of metabolites from $S$. pyridomyceticus recombinant

22 strains. The detection of each strain was individually scanned by using MS/MS fragmentation patterns

23 of $m / z 409$ for pyridomycin and 411 for pyridomycin B. 


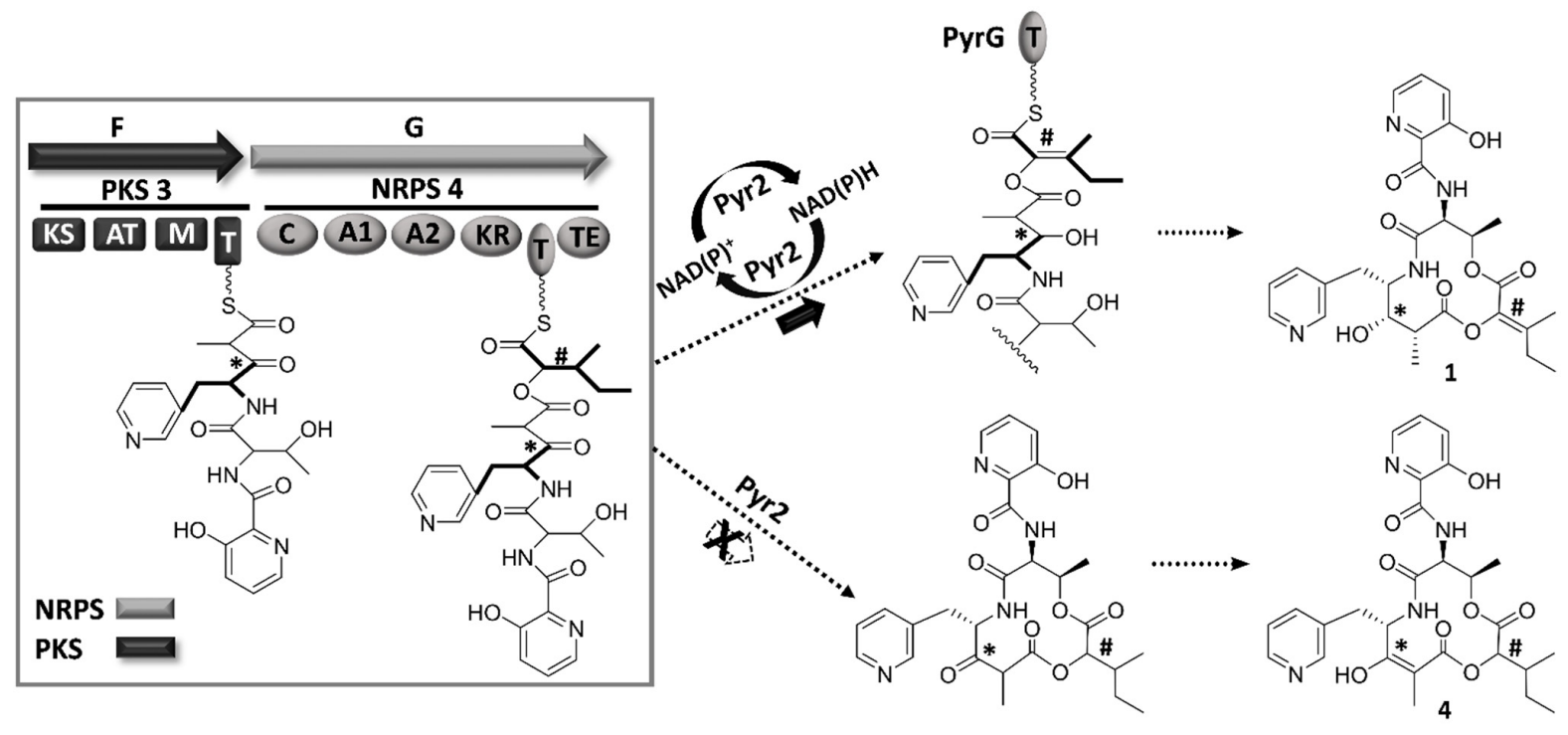

26 Figure 5. Proposed biosynthesis of pyridomycin supported by the isolation of pyridomycin B 